\title{
Comparación de ACV de un refuerzo estructural de plancha de acero y de un refuerzo de lámina de fibra de carbono ambos adheridos con epoxi
}

\author{
B. Palacios-Muñoz ${ }^{1}$, B. López-Mesa ${ }^{2}$, L. Gracia-Villa ${ }^{1}$ \\ ${ }^{1}$ Afiliación: Grupo de Biomateriales (GBM) \\ Instituto de Investigación en Ingeniería de Aragón (I3A) \\ Universidad de Zaragoza, Mariano Esquillor s/n, 50018, Zaragoza, Spain. \\ Tel. +34-976762707, e-mail: bpalacios@unizar.es \\ ${ }^{2}$ Afiliación: Unidad Predepartamental de Arquitectura, Universidad de Zaragoza
}

\begin{abstract}
Este trabajo presenta una comparación de impacto medioambiental, aplicando la metodología de Análisis de Ciclo de Vida (ACV), de dos refuerzos estructurales a flexión adheridos con resina epoxi: un refuerzo de plancha acero, de utilización tradicional, y un refuerzo con fibra de carbono, uno de los más utilizados actualmente.
\end{abstract}

\section{Introducción}

Para alcanzar los ambiciosos objetivos energéticos fijados por la Unión Europea a través de diferentes normativas (2012/27/UE, 2010/31/UE, 2002/91/CE) es indispensable la atención al sector de la edificación, responsable (incluyendo únicamente a edificios residenciales y de servicios) del 35\% de las emisiones equivalentes de CO2 de la UE en 2011 [1].

Analizar este impacto de manera rigurosa implica considerar todas las fases del proceso edificatorio “desde la cuna a la tumba”: construcción, uso, rehabilitación/es y demolición final. Esto es lo que se conoce como Análisis de Ciclo de Vida (ACV).

De acuerdo con la bibliografía, la mayor parte de los estudios de ACV aplicados a edificación (incluida la rehabilitación) ponen su énfasis en el impacto de la fase de construcción y uso, pero no incorporan el debido a las intervenciones de reparación y/o intervención en los sistemas del edificio como la estructura [1]. La estructura, por los materiales y procesos que involucra, supone un importante porcentaje de este impacto: alrededor de un $11.7 \%$, sin considerar rehabilitaciones, según [2].

A lo largo de la vida útil de un edificio es probable que sea necesaria la intervención sobre el mismo debido a cuestiones como cambio de uso o posibles lesiones. Atendiendo a diversos datos estadísticos como [3], [4], cerca del $6 \%$ de las lesiones producidas en edificaciones en los 10 primeros años son estructurales, porcentaje superior si consideramos las lesiones a largo plazo. La mayor parte de ellas se presentan en elementos a flexión (forjados + vigas). Los impactos de estas intervenciones deben ser incluidas en el ACV. Este trabajo supone una contribución al respecto.

\section{Material y métodos}

Para la consecución del objetivo señalado, se parte de la necesidad hipotética de reforzar una viga de hormigón armado a flexión, que cumple determinados requisitos. Posteriormente se realizan dos fases sucesivas: dimensionado de ambos refuerzos para prestaciones equivalentes y $\mathrm{ACV}$ de los mismos.

\section{Fase A: Dimensionado de ambos refuerzos}

La metodología seguida para el cálculo del refuerzo se describe a continuación.

A.1) Dimensionado de la sección del refuerzo. Para el dimensionado del refuerzo es necesario incorporar al cálculo la deformación ya existente en la viga, producida por las cargas permanenetes, pues no se considera la descarga completa del elemento por cuestiones prácticas. Por ello, este dimensionado comprende dos etapas sucesivas.

La primera, busca la obtención de las deformaciones del hormigón y de la armadura debidas a las acciones permanentes sobre la viga sin reforzar en valor de servicio. Esto se obtiene mediante la aplicación sucesiva de las ecuaciones de compatibilidad, comportamiento y equilibrio, y la resolución del sistema de ecuaciones no lineal.

La segunda, con la que se obtiene el dimensionado del refuerzo, precisa la obtención del diagrama de interacción de esfuerzo axil y momento flector de la viga reforzada, incorporando las deformaciones 
iniciales y considerando las acciones y propiedades de los materiales en valor de cálculo.

A.3) Cálculo de la longitud del refuerzo y la longitud de anclaje. La longitud del refuerzo se obtiene a partir de la envolvente de momentos flectores y la longitud de anclaje aplicando el modelo A, incorporado en el código del CEB-FIB.

\section{A.4) Comprobación de tensiones rasantes.}

Los materiales del refuerzo poseen propiedades convencionales.

\section{Fase B: ACV de los refuerzos analizados}

La metodología seguida para el ACV comparativo es la relacionada en la norma ISO 14040, utilizando el software SimaPro v.7.0. Las fases incluidas en la norma son las siguientes: definición de objetivos y alcance; análisis de inventario; análisis de impacto (utilizando en este trabajo los indicadores Ecoindicator 99 (E) V2.08 y IPCC 2007 GWP 100a V1.02) e interpretación de resultados.

Se incluyen los impactos producidos por todos los procesos y materiales involucrados hasta la completa ejecución, excepto los impactos presentes en ambos esfuerzos, por tratarse de un ACV compartivo.

\section{Resultados}

Los resultados obtenidos en la fase A se reflejan en la Tabla 1 y en la Fig. 1.

Respecto de la fase $\mathrm{B}$, cabe señalar que aunque la producción de $1 \mathrm{~kg}$ de lámina de fibra de carbono conlleva un impacto ambiental muy superior a la obtención de $1 \mathrm{~kg}$ de acero laminado (del orden de 9 veces más), en el ACV comparado de ambas intervenciones, el refuerzo de acero obtiene un resultado más desfavorable debido a que las altas prestaciones mecánicas de la fibra de carbono permiten una elevada optimización de la cantidad de material.

\section{Tabla 1 Dimensionado de los refuerzos}

\begin{tabular}{lll}
\hline Variable & Refuerzo acero & Refuerzo fibra \\
\hline ancho & $50 \mathrm{~mm}$ & $13 \mathrm{~mm}$ \\
espesor & $1.5 \mathrm{~mm}$ & $1.2 \mathrm{~mm}$ \\
largo & $1816 \mathrm{~mm}$ & $1711 \mathrm{~mm}$
\end{tabular}

\section{Conclusiones}

Desde el punto de vista mecánico, la utilización de láminas de fibra de carbono permite obtener prestaciones equivalentes utilizando un $19 \%$ menos de material y con una densidad 5 veces menor aproximadamente. Desde un punto de vista medioambiental, a pesar de que el impacto de fabricación de $1 \mathrm{~kg}$ de lámina de fibra de carbono es superior al de $1 \mathrm{~kg}$ de acero laminado, la posibilidad de reducir la cantidad de material empleado gracias a las buenas propiedades mecánicas obtenidas, resulta en un impacto final mucho menor (unas 4 veces menos emisiones de $\mathrm{CO}_{2}$ eq. que el refuerzo de acero según el indicador IPCC GWP 100a).

Los resultados de este trabajo suponen una contribución a la incorporación del impacto de las intervenciones estructurales en el ACV global de un edificio así como una herramienta de orientación a la hora de seleccionar la alterntativa más adecuada.

\section{REFERENCIAS}

[1] VILCHES, A, GARCIA-MARTINEZ, A and SANCHES-MONTA, B. Life cycle assessment (LCA) of building refurbishment: A literature review. Energy and Buildings. 2017, 135, 286-301. Available from: doi: 10.1016/j.enbuild.2016.11.042

[2] DOBBELSTEEN, AAJF, VAN DEN, LINDEN, AC and VAN DER RAVESLOOT, CM. Defining the reference for environmental performance. In: Proceedings of the InternationalConference on Advances in Building Technology, Vol. II, Hong Kong, China, 2002. pp. 1509-16.

[3] CANSADO CARRETERO, A and MORENO CANSADO, MJ. Análisis estadístico nacional sobre patologías en la edificación (II) - Resumen. Fundación MUSAAT, 2013, Informe Técnico.

[4] GRUPO ESPAÑOL DEL HORMIGÓN ARMADO (GEHO). Encuesta sobre patología de estructuras de hormigón. Grupo Español de hormigón - Comisión GT IV - Boletín nº10, 1992.

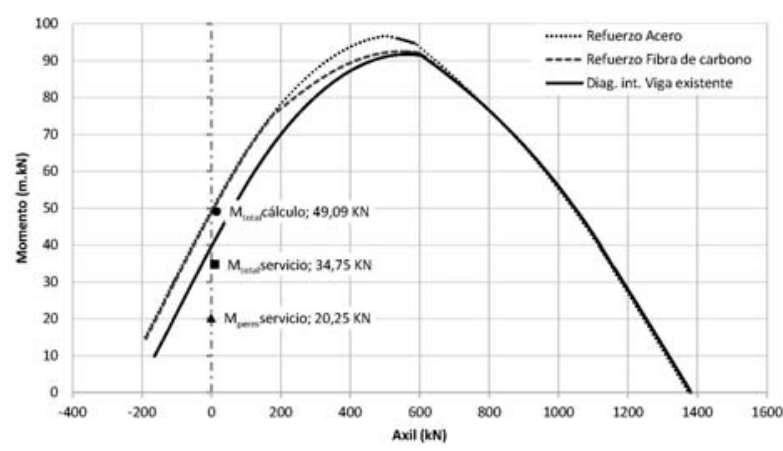

Fig. 1 Diagrama interacción sección reforzada 